\title{
Spectrum of Novel Hereditary Hemorrhagic Telangiectasia Variants in an Austrian Patient Cohort
}

\author{
Martin Koenighofer ${ }^{1}$ (1) $\cdot$ Thomas Parzefall $^{1}$ (1) $\cdot$ Alexandra Frohne ${ }^{2}$ (1) $\cdot$ Matthew Allen $^{2}$ (1) $\cdot$ Ursula Unterberger $^{2}$ (1) \\ Franco Laccone ${ }^{3}$ (i) $\cdot$ Christian Schoefer ${ }^{2}$ (1) $\cdot$ Klemens Frei $^{1}$ (D) $\cdot$ Trevor Lucas $^{2}$ (1) \\ ${ }^{I}$ Department of Otorhinolaryngology, Head and Neck Surgery, ${ }^{2}$ Center for Anatomy and Cell Biology, Department for Cell and Developmental \\ Biology, and ${ }^{3}$ Institute of Medical Genetics, Medical University of Vienna, Vienna, Austria
}

Objectives. Hereditary hemorrhagic telangiectasia $(\mathrm{HHT})$ is a rare autosomal dominant genetic disorder characterized by pathogenic blood vessel development and maintenance. HHT type 1 (HHT1) and type 2 (HHT2) are caused by variants in endoglin (ENG) and activin receptor-like kinase-1 (ACVRL1), respectively. The aim of this study was to identify the spectrum of pathogenic variants in ENG and ACVRL1 in Austrian HHT families.

Methods. In this prospective study, eight Austrian HHT families were screened for variants in ENG and ACVRL1 by polymerase chain reaction amplification and sequencing of DNA isolated from peripheral blood.

Results. Heterozygous variants were identified in all families under study. HHT1 was caused by a novel c.816+1G >A splice donor variant, a novel c.1479C $>$ A nonsense (p.Cys493X) variant and a published c.1306C $>$ T nonsense (p.Gln436X) variant in ENG. Variants found in ACVRL1 were novel c.200G >C (p.Arg67Pro) and known c.772G >A (p.Gly258Ser) missense variants in highly conserved residues, a known heterozygous c.100dupT frameshift (p.Cys34Leufs*4) and the known c.1204G > A missense (p.Gly402Ser) and c.1435C > T nonsense (p.Arg479X) variants as causes of HHT2.

Conclusion. Novel and published variants in ENG (37.5\%) and ACVRL1 (62.5\%) were exclusively identified as the cause of HHT in an Austrian patient cohort. Identification of novel causative genetics variants should facilitate the development of tailored therapeutical applications in the future treatment of autosomal dominant HHT.

Keywords. Telangiectasia; Hereditary Hemorrhagic; Endoglin; Activin Receptor-Like Kinase-1

\section{INTRODUCTION}

Hereditary hemorrhagic telangiectasia (HHT) is an incurable progressive genetic disorder inherited in an autosomal dominant pattern that manifests as dysfunctions in vasculogenesis, angiogenesis and maintenance of the normal vasculature. Distinctions are made between small dermal and mucosal lesions called telangiectasias and visceral arteriovenous malformations, which

\footnotetext{
- Received March 4, 2019

Revised May 1, 2019

Accepted May 22, 2019

- Corresponding author: Klemens Frei

Department of Otorhinolaryngology, Head and Neck Surgery, Medical University of Vienna, AKH-8J,Währinger Gürtel 18-20, A-1090, Vienna, Austria

Tel: +43-1-40400-33480, Fax: +43-1-40400-64510

E-mail: klemens.frei@meduniwien.ac.at
}

are larger and mostly found in the liver, lungs and brain. In HHT, blood vessel malformations occur whereby dilation and deterioration of capillaries can result in a total loss of the capillary bed leading to a shunt between arteries and veins [1]. Since deformed vessels are less stable towards mechanical stress, epistaxis and visceral hemorrhage are cardinal symptoms of HHT together with visible telangiectasias on the skin and oral mucosa [2].

The worldwide prevalence of HHT is estimated at 1:5,000/ 8,000 and it is listed as an orphan disease by the U.S. Office of Rare Diseases (Genetic and Rare Diseases Information Center) and European Orphanet (classification number: ORPHA774). The incidence of HHT can be significantly higher in specific regions such as Curaçao and Bonaire $(1: 1,331)$ or in the Haut-Jura region $(1: 2,351)$ of France [3]. In 2000, the Curaçao criteria were introduced, which define the clinical diagnostic features of HHT as telangiectasia, recurrent epistaxis, visceral lesions, and a posi-

Copyright @ 2019 by Korean Society of Otorhinolaryngology-Head and Neck Surgery

This is an open-access article distributed under the terms of the Creative Commons Attribution Non-Commercial License (http://creativecommons.org/licenses/by-nc/4.0)

which permits unrestricted non-commercial use, distribution, and reproduction in any medium, provided the original work is properly cited. 
tive family history. If three criteria are met, diagnosis is regarded as confirmed [4,5]. Due to the progressive course of HHT, the application of these criteria is reduced in children. At present, there is no cure for HHT, and treatment is confined to iron supplementation and transfusions to treat anemia and blood loss and reducing the frequency of epistaxis by laser therapy or skin grafting $[2,6,7]$.

Genetic testing of HHT patients permits a subclassification into HHT types 1-5. The majority of HHT cases are HHT type 1 (HHT1; online mendelian inheritance in man [OMIM]: \#187300) or type 2 (HHT2; OMIM: \#600376), caused by mutations in the endoglin $(E N G)$ or activin receptor-like kinase-1 (ACVRL1) genes, respectively [8]. Although the pathogenesis is similar, the rate of pulmonary and cerebral involvement may be higher in HHT1 [9]. Heterozygous mutations in ACVRL1 and ENG support a model of haploinsufficiency for HHT1 and HHT2 [6]. Mutations in ACVRL1 and ENG are globally relevant for the development of HHT in European, African and Asian populations $[1,3,4]$.

Two loci on chromosomes 5 and 7 are associated with the development of HHT3 (OMIM: \#601101) and HHT4 (OMIM: \#610655), respectively [10,11], whereas HHT5 (OMIM: \#615506) is caused by mutations in GDF2 on chromosome 10, coding for bone morphogenetic protein 9 (BMP9). Alterations in the SMAD family member 4 gene (SMAD4) can cause a combined syndrome of HHT and juvenile polyposis (OMIM: \#175050) [12].

The ENG, ACVRL1 and SMAD4 proteins are all components of the transforming growth factor beta (TGF- $\beta$ ) signaling pathway, which is pivotal for vascular development and endothelial cell maintenance [13]. The TGF- $\beta$ superfamily is a group of regulatory proteins that includes TGF- $\beta$ proteins and BMP proteins. In the canonicalTGF- $\beta$ pathway, signal transduction is performed by receptor complexes with serine/threonine kinase activity and SMAD transcription factors. Binding of a TGF- $\beta$ ligand induces a type II TGF- $\beta$ receptor (TGFBR2) dimer to recruit a type I TGF- $\beta$ receptor (TGFBR1) dimer resulting in a heterotetrameric receptor complex, which leads to phosphorylation of a receptorregulated-(R)SMAD protein, formation of an (R-)SMAD complex with a common mediator (Co-)SMAD and transport of the complex to the nucleus where transcription is modulated. In endothelial cells, ENG and ACVRL1 promote or inhibit angiogen-

\section{H}

- Novel variants in hereditary hemorrhagic telangiectasia (HHT) patients have been identified.

- Predicted pathomechanisms in novel variants show loss of function.

- HHT variants could facilitate tailored therapeutical applications. esis depending on receptor complex composition. ACVRL1 serves as aTGFBR1, which in complex with a TGFBR2 mediates effects via R-SMAD1/5/8, resulting in the expression of genes associated with angiogenesis and cell proliferation.

ACVRL1 is a 503-residue TGF- $\beta$ type I serine-threonine kinase receptor with a single transmembrane domain expressed as a dimer on the surface of endothelial cells. Transcribed from 9 coding exons at 12q13.13, ACVRL1 has a 21-residue signal peptide and a short 96-residue cysteine-rich extracellular domain for interaction with TGF- $\beta$ and BMP9 [14]. Residues within the extracellular domain govern specificity for BMP ligands [15]. ACVRL1 has an intracellular glycine- and serine-rich domain, phosphorylation of which by a type II receptor activates the C-terminal kinase domain to activate SMAD phosphorylation [13].

$E N G$ is transcribed from 15 exons at $9 \mathrm{q} 34.11$ and encodes a 658-residue transmembrane glycoprotein expressed as a proangiogenic disulfide bridge-linked homodimer [16]. ENG has a large extracellular domain encompassing 586 amino acids that form two zona pellucida domains and an "orphan" domain, which bears no homology to known proteins. The zona pellucida domain is thought to be important for dimerization, whereas the orphan domain seems to play a role in ligand recognition. The residues from position 1 to 26 serve as a signal peptide and are removed during processing. The ectodomain of ENG is highly glycosylated and is a dome-like structure of antiparallel arranged monomers shaped as a cavity [17]. The 47-residue serine-/threonine-rich intracellular region of L (large)-ENG contains a zona pellucida domain-binding motif that plays a role in modulating downstream signaling. Certain serine and threonine residues are constitutively phosphorylated. TGFBR1 and TGFBR2 interact with the cytoplasmic and zona pellucida-C domains of ENG [18]. A second isoform S-ENG (short) is generated by alternative splicing and has an intracellular region of only 14 residues [19]. Although ENG can bind some ligands such as BMP9 and BMP10 independently, it is primarily an auxiliary receptor that modulates signaling by affecting ligand binding in a complex with a TGFBR1 such as ACVRL1. A soluble form of $E N G$ is generated by extracellular domain juxtamembrane metalloproteinase-14 cleavage. The soluble form of ENG can capture ligand [20] and is considered anti-angiogenic [13]. In this study, index patients from eight Austrian families diagnosed with HHT were screened for mutations in ENG and ACVRL1 to determine HHT1 and HHT2 status.

\section{MATERIALS AND METHODS}

Individuals suffering from HHT diagnosed according to Curaçao criteria [5] were recruited from the Department of Otorhinolaryngology, University Hospital of Vienna. This study was approved by the Vienna Ethics Committee (No. 1328/2014) and 
compliant with the ethical standards due to the Declaration of Helsinki. Informed consent has been taken from any participant of this study prior to inclusion. Complete medical histories and otorhinolaryngological status of all individuals were examined. Clear autosomal dominant inheritance of HHT was observed in seven families.

DNA was extracted from peripheral blood with the Invisorb blood universal kit 1000 (STRATEC Molecular, Berlin, Germany). The full coding sequences of ENG (GenBank accession NM_001114753.2) and ACVRL1 (NM_000020.2) were amplified and sequenced as described previously with some modifications with primers [21] in a reaction mixture containing $200 \mu \mathrm{M}$ dNTPs, 2 mM MgCl 2,20 mM Tris- $\mathrm{HCl}$ (pH 8), 50 mM KCl, 5\% DMSO and $1 \mathrm{U}$ Platinum Taq polymerase (Invitrogen, Carlsbad, CA, USA) for 40 cycles of amplification with annealing at primer specific temperatures for 40 seconds, extension at $72^{\circ} \mathrm{C}$ for 60 seconds, denaturation at $94^{\circ} \mathrm{C}$ for 40 seconds and end extension at $72^{\circ} \mathrm{C}$ for 7 minutes in a GeneAmp PCR System 9700 thermal cycler (PE Applied Biosystems, Foster City, CA, USA). PCR products were separated on $1.5 \%$ agarose gels containing
$0.5 \mu \mathrm{g} / \mathrm{mL}$ ethidium bromide at $1 \mathrm{~V} / \mathrm{cm}$ and sequenced on an ABI sequencer. Results were compared to the wild type locus sequence using the NCBI interface (https://blast.ncbi.nlm.nih. gov/Blast.cgi?PAGE_TYPE=BlastSearch\&BLAST_SPEC $=$ blast2seq\&LINK_LOC=align2seq). Novel mutations were identified by literature searches and by reference to the ENG and ACVRL1 mutation databases at the University of Utah (http://arup.utah. edu/database/HHT). All identified variants were registered at ClinVar (SCV000346037-44). Sequences are ClustalW multiple sequence alignments (http://webcitation.org) with Homo sapiens (UniProt P37023), Pan troglodytes (H2Q5Y5), Canis lupus familiaris (E2R174), Mus musculus (Q91YV1), Rattus norvegicus (P80203), Gallus (XP_003643441.2), Xenopus tropicalis (F6XT30) and Danio rerio (Q498X0) sequences depicted with BoxShade (http://www.ch.embnet.org/software/BOX_form. html). The severity of missense mutations was predicted with Polyphen-2 (http://genetics.bwh.harvard.edu/pph2) and PROVEAN (http://provean.jcvi.org/index.php). Global allele frequencies were determined with the gnomAD browser (http://gnomad.broadinstitute.org) [22].

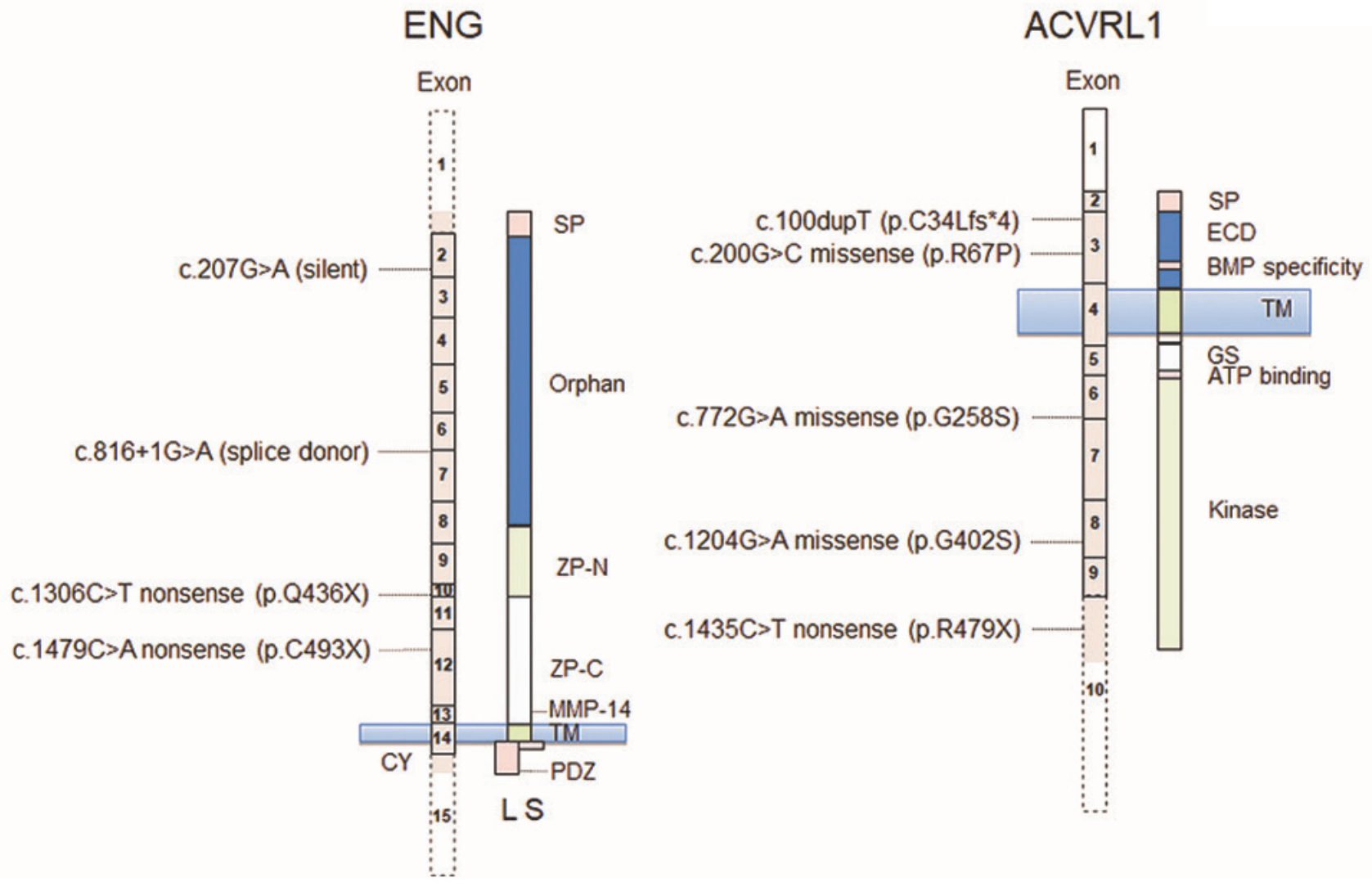

Fig. 1. Schematic diagram of variants identified in relation to exon and protein domains of endoglin (ENG) and activin receptor-like kinase-1 (ACVRL1). Signal peptides (SP), transmembrane domains (TM), ENG N- and C-terminal zona pellucida (ZP) domains, the juxtamembrane metalloproteinase-14 (MMP-14) cleavage site to produce soluble ENG, generation of short (S) and long (L) membrane bound forms by differential splicing within exon 14 in the cytoplasmic $(C Y)$ domain, the zona pellucida binding motif in L-ENG together with the region of the ACVRL1 extracellular domain (ECD) determining specificity for bone morphogenetic protein (BMP) and cytoplasmic glycine- and serine-rich (GS) region (dotted exons not drawn to scale) are illustrated. ATP, adenosine triphosphate. 


\section{RESULTS}

We identified three unpublished, five known and one silent variant in ENG and ACVRL1 (Fig. 1) in eight unrelated, nonconsanguineous families in Austria. In total, two novel variants and one known variant were identified in ENG. In one family (Fig. 2A) an unpublished intronic transition (rs111471193; ClinVar SCV000346037), listed in the ARUP database, was found (between exons 6 and $7 ;$ c. $816+1 \mathrm{G}>\mathrm{A}$ ) that mutates the guanine residue in the highly conserved GU splice donor motif. A novel c.1479C > A nonsense p.Cys493X (SCV000346038) transversion in exon 12 was identified in a second family (Fig. 2B) causing the replacement of cysteine with an opal stop codon at p.493. The proband also suffered from a cerebral arteriovenous malformation, which was embolized with computed tomographic angiography. In the third family, a heterozygous c.1306C $>$ T nonsense (p.Gln436X; SCV000346039) transition in exon 10 was found (Supplementary Fig. 1) that has been described previously [23].

In total, one unpublished and three known variants were identified in ACVRL1. A heterozygous insertion of a thymidine after c.100dupT (Supplementary Fig. 2) in exon 3 (p.Cys34Leufs*4; HGMD CI111588; SCV000346042) was identified in one family that is listed in the ARUP database [24]. The index patient had pancreatic, hepatic and pulmonic arteriovenous malformations. A novel heterozygous c.200G $>C$ missense transversion (p.Arg67Pro; SCV000346040) was found in the extracellular domain of ACVRL1 (Fig. 3) in another family. In the third family a c.772G > A (p.Gly258Ser; SCV000346041) missense transition in a highly conserved residue was found that is pending classification on the ARUP database. The mutation has been described previously [25] and is positioned at the distal end of exon 6 neighboring an atypical GC splice donor consensus (Supplementary Fig. 3). The index patient had pancreatic and hepatic arteriovenous malformations. Two other known variants found in two other families are the missense transition c.1204G $>$ A (p.Gly402Ser; SCV000346043) $[10,26]$ in exon 8 in a highly conserved residue (Supplementary Fig. 4) and the c.1435C $>$ T (p.Arg479X; SCV000346044) nonsense transition in exon 10 (Supplementary Fig. 5) [27]. A single example of c.1435C $>\mathrm{T}$ is listed in the gnomAD database in a Latino subject (global 1/ 246,$\left.214 ; 4.1 \times 10^{-6}\right)$.

In addition to pathogenic variants, the c.207G $>$ A transition in exon 2 of $E N G$ was found in two families together with the known pathogenic $E N G$ heterozygous nonsense transition c.1306C $>\mathrm{T}$ [23] (Supplementary Fig. 1) and the known ACVRL1 missense variant c.772G $>$ A identified in this study (Supplementary Fig. 3).

\section{DISCUSSION}

In this study, nonsense, frameshift, splice donor and missense variants in conserved residues were identified. Late onset dis-

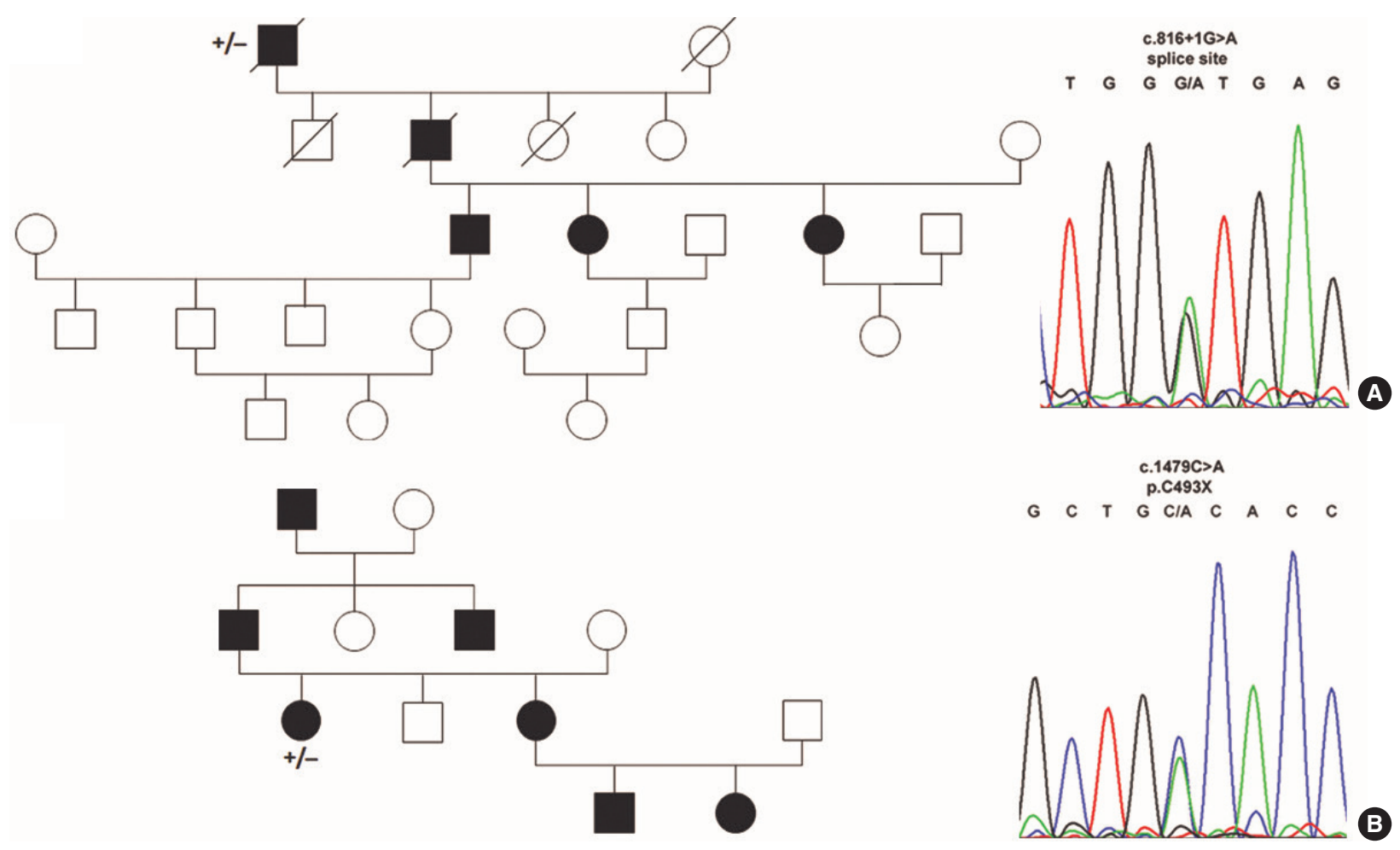

Fig. 2. Endoglin (ENG) mutations identified in families suffering from hereditary hemorrhagic telangiectasia. (A) A heterozygous ENG c.816+1G> A splice site variant (B) and a c.1479C >A nonsense (p.C493X) mutation were identified in the proband analyzed (+/-) by Sanger sequencing. 

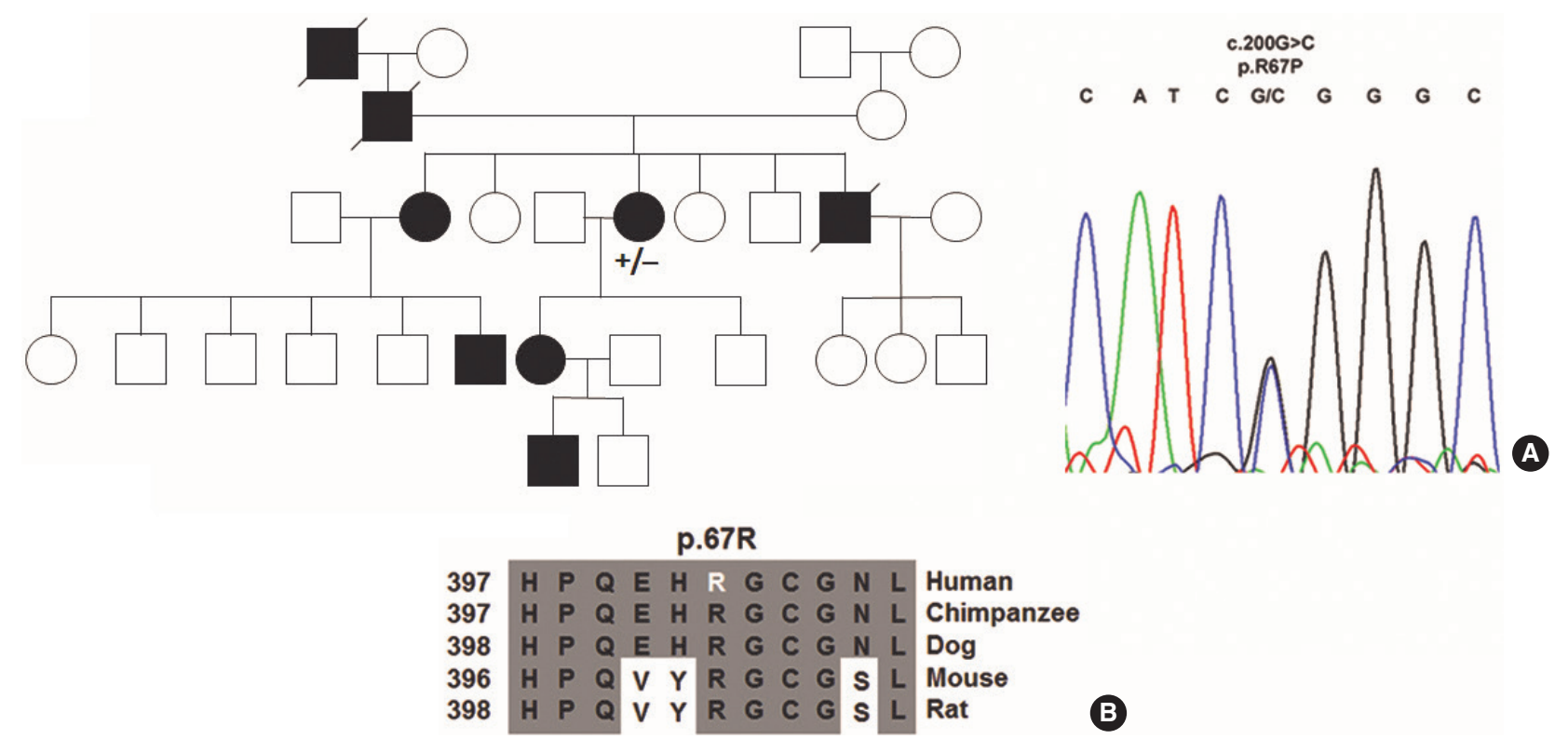

Fig. 3. Novel activin receptor-like kinase-1 (ACVRL 1) mutation identified in families suffering from hereditary hemorrhagic telangiectasia. (A) A c.200G $>$ C missense (p.R67P) mutation were identified in the proband analyzed (+/-) by Sanger sequencing. (B) Cross species sequence alignments show the arginine residue at p.67 is highly conserved in mammals.

ease variants are commonly listed in allele frequency databases of healthy populations. The pathogenic variants identified are exceptionally rare and only a single listing of c.1435C $>$ T worldwide is represented in the gnomAD allele frequency database [22]. Three of the mutations identified are unpublished in European and American populations to date and the pathogenic mechanisms leading to HHT1 and HHT2 can be predicted. Regardless of mutation, onset according to Curaçao criteria was in early adolescence in all cases.

The ENG transition (c.816+1G>A) mutates the highly conserved GU intron 6 splice donor motif. If intron retention were to occur, translation from the mRNA would terminate at an opal stop codon at c.816+(88-90). The 29-residue peptide sequence "MSCAQLPGHKTQTPNLWIREVSWKGEPPS" which has limited homology to a hypothetical putative enterobacterial transcription regulator DUF1323 (pfam07037) would be predicted to be translated distal to p.Trp272 within the orphan domain. A novel ENG variant identified is a nonsense c.1479C > A (p.Cys493X) variant predicted to cause synthesis of truncated ENG comprising only the N-terminal 437 residues [28]. It would therefore be expected that a translated peptide comprising the signal peptide, the orphan domain but only a fragment of the zona pellucida- $\mathrm{C}$ domain would be secreted, lacking the transmembrane and intracellular domains. Cysteine residues between p.330 and p.412 may cause dimerization between wildtype ENG and p.Cys493X ENG [28]. The truncated ENG would also not be subject to matrix MMP-14 cleavage in the juxtamembrane region of the zona pellucida-C domain at residues p.586-587 [19]. Amino acids within the zona pellucida- $\mathrm{C}$ domain between positions $\mathrm{p} .437-$
558 control interactions with TGFBR1 and TGFBR2, suggesting that p.Cys493X would disrupt TGF- $\beta$ signaling modulation. This gene product, however, contains almost all domains present in a soluble form of ENG. Since the orphan domain is sufficient for binding BMP9/10, these ligands could be sequestered from their receptors by the mutant peptide to affect downstream pathways. Therefore, in addition to haploinsufficient effects caused by the absence of membrane bound ENG, p.Cys493X ENG could also lead to sequestration of circulating BMP.

The c.100dupT insertion in ACVRL1 (http://arup.utah.edu/ database/HHT) is predicted to create an opal stop codon 11 residues after p.Thr33 causing translation to be terminated after 3 ("LHV") residues (p.Cys34Leufs*4). The novel ACVRL1 missense variant c.200G >C (p.Arg67Pro) is predicted by bioinformatics to be either possibly damaging (Polyphen-2-score, 0.95; sensitivity, 0.79; specificity, 0.95) or deleterious (PROVEAN: -3.393). Variants in codon 67 have been linked previously to the development of HHT2. The c.200G > A (p.Arg67Gln) alteration [29] has been described previously [1] and c.199C $>\mathrm{T}$ (p.Arg67Trp) has been identified in an Italian family [30]. The extracellular domains of ACVRL1 and ACTRIIB have been crystallized in a complex with BMP9 (PDB;4FAO) [15]. The ACVRL1 extracellular domain (p.22-118) contains $5 \beta$-pleated sheets $(\beta)$ numbered from the cleaved N-terminus (p.Arg67 is positioned in $\beta 4$ ). Whereas the adjacent $\mathrm{p}$.His66 residue forms a hydrophobic pocket at the BMP9 interface, the charged arginine residue at p.67 builds a salt bridge with p.Glu65 and charge-dependent interactions with the extracellular domain C-terminal residues p.Asn96, p.His97 and p.Val99. Replacement of cationic 
charged p.Arg67 with a neutral Pro residue could potentially interrupt these reactions similar to predictions for the uncharged glycine or tryptophan residue substitutions [15]. In addition, it has been reported that p.Arg67Gln is retained in the endoplasmatic reticulum [31].

Although silent, inheritance of the common c.207G >A (rs11545664; p.Leu69X) variant (gnomAD European non-Finnish allele frequency 0.104) in ENG may be weakly and nonsignificantly associated with sporadic brain arteriovenous malformations and reduces predicted binding scores for the splicing factor SRp40 [32]. In this study, the silent c.207G>A ENG variant was identified in patients (data not shown) also bearing the c.1306C > T ENG variant (no arteriovenous malformation development) and the c.772G $>$ A ACVRL1 variant (pancreatic and hepatic arteriovenous malformation development).

In this Austrian study cohort, we determined the frequency of HHT1 and HHT2 as $37.5 \%$ and $62.5 \%$, respectively. Expansion of the molecular diagnostic variant spectrum in HHT should accelerate the development of novel variant-specific therapies in the treatment of autosomal dominant HHT.

\section{CONFLICT OF INTEREST}

No potential conflict of interest relevant to this article was reported.

\section{ACKNOWLEDGMENTS}

This study was supported by funds of the medical-scientific fund of the mayor of Vienna (Project No. 17107), Vienna, Austria.

We would like to thank the families for their cooperation.

\section{ORCID}

Martin Koenighofer

https://orcid.org/0000-0002-1380-9170

Thomas Parzefall https://orcid.org/0000-0002-7817-5789

Alexandra Frohne

Matthew Allen https://orcid.org/0000-0001-6641-4001

Ursula Unterberger

Franco Laccone https://orcid.org/0000-0001-9782-5567 https://orcid.org/0000-0003-2233-1006 https://orcid.org/0000-0001-9466-6441

Christian Schoefer https://orcid.org/0000-0001-9570-5692

Klemens Frei https://orcid.org/0000-0001-6301-2445

Trevor Lucas https://orcid.org/0000-0002-5114-8035

\section{AUTHOR CONTRIBUTIONS}

Conceptualization: TL, KF. Data curation: MK, TP, AF, MA, FL. Formal analysis: MK, AF, TL. Funding acquisition:TL, KF. Meth- odology: MK, AF, UU, FL, CS, TL, KF. Project administration: TL, KF. Visualization: TL, MK. Writing - original draft: MK, TL. Writing - review \& editing: all authors.

\section{SUPPLEMENTARY MATERIALS}

Supplementary materials can be found via https://doi.org/10. 21053/ceo.2019.00304.

\section{REFERENCES}

1. Schulte C, Geisthoff U, Lux A, Kupka S, Zenner HP, Blin N, et al. High frequency of ENG and ALK1/ACVRL1 mutations in German HHT patients. Hum Mutat. 2005 Jun;25(6):595.

2. McDonald J, Pyeritz RE. GeneReviews: hereditary hemorrhagic telangiectasia [Internet]. Seattle, WA: University of Washington; 2000 [cited 2019 May 25]. Available from: https://www.ncbi.nlm.nih.gov/ books/NBK1351/.

3. Westermann CJ, Rosina AF, De Vries V, de Coteau PA. The prevalence and manifestations of hereditary hemorrhagic telangiectasia in the Afro-Caribbean population of the Netherlands Antilles: a family screening. Am J Med Genet A. 2003 Feb;116(4):324-8.

4. Lee ST, Kim JA, Jang SY, Kim DK, Do YS, Suh GY, et al. Clinical features and mutations in the ENG, ACVRL1, and SMAD4 genes in Korean patients with hereditary hemorrhagic telangiectasia. J Korean Med Sci. 2009 Feb;24(1):69-76.

5. Shovlin CL, Guttmacher AE, Buscarini E, Faughnan ME, Hyland $\mathrm{RH}$, Westermann CJ, et al. Diagnostic criteria for hereditary hemorrhagic telangiectasia (Rendu-Osler-Weber syndrome). Am J Med Genet. 2000 Mar;91(1):66-7.

6. Abdalla SA, Letarte M. Hereditary haemorrhagic telangiectasia: current views on genetics and mechanisms of disease. J Med Genet. 2006 Feb;43(2):97-110.

7. Begbie ME, Wallace GM, Shovlin CL. Hereditary haemorrhagic telangiectasia (Osler-Weber-Rendu syndrome): a view from the 21st century. Postgrad Med J. 2003 Jan;79(927):18-24.

8. Sadick H, Hage J, Goessler U, Stern-Straeter J, Riedel F, Hoermann $\mathrm{K}$, et al. Mutation analysis of "Endoglin" and "Activin receptor-like kinase" genes in German patients with hereditary hemorrhagic telangiectasia and the value of rapid genotyping using an allele-specific PCR-technique. BMC Med Genet. 2009 Jun;10:53.

9. LetteboerTG, Mager JJ, Snijder RJ, Koeleman BP, Lindhout D, Ploos van Amstel JK, et al. Genotype-phenotype relationship in hereditary haemorrhagic telangiectasia. J Med Genet. 2006 Apr;43(4):371-7.

10. Bayrak-Toydemir P, McDonald J,Akarsu N,Toydemir RM, Calderon F, Tuncali T, et al. A fourth locus for hereditary hemorrhagic telangiectasia maps to chromosome 7. Am J Med Genet A. 2006 Oct; 140(20):2155-62.

11. Cole SG, Begbie ME, Wallace GM, Shovlin CL. A new locus for hereditary haemorrhagic telangiectasia (HHT3) maps to chromosome 5. J Med Genet. 2005 Jul;42(7):577-82.

12. Gallione CJ, Repetto GM, Legius E, Rustgi AK, Schelley SL, Tejpar S, et al. A combined syndrome of juvenile polyposis and hereditary haemorrhagic telangiectasia associated with mutations in MADH4 (SMAD4). Lancet. 2004 Mar;363(9412):852-9.

13. Mahmoud M, Upton PD, Arthur HM. Angiogenesis regulation by TGF $\beta$ signalling: clues from an inherited vascular disease. Biochem Soc Trans. 2011 Dec;39(6):1659-66.

14. Mahlawat P, Ilangovan U, Biswas T, Sun LZ, Hinck AP. Structure of 
the Alk1 extracellular domain and characterization of its bone morphogenetic protein (BMP) binding properties. Biochemistry. 2012 Aug;51(32):6328-41.

15. Townson SA, Martinez-Hackert E, Greppi C, Lowden P, Sako D, Liu $\mathrm{J}$, et al. Specificity and structure of a high affinity activin receptor-like kinase 1 (ALK1) signaling complex. J Biol Chem. 2012 Aug;287(33): 27313-25.

16. Alt A, Miguel-Romero L, Donderis J,Aristorena M, Blanco FJ, Round A, et al. Structural and functional insights into endoglin ligand recognition and binding. PLoS One. 2012;7(2):e29948.

17. Llorca O, Trujillo A, Blanco FJ, Bernabeu C. Structural model of human endoglin, a transmembrane receptor responsible for hereditary hemorrhagic telangiectasia. J Mol Biol. 2007 Jan;365(3):694-705.

18. Guerrero-Esteo M, Sanchez-Elsner T, Letamendia A, Bernabeu C. Extracellular and cytoplasmic domains of endoglin interact with the transforming growth factor-beta receptors I and II. J Biol Chem. 2002 Aug;277(32):29197-209.

19. Hawinkels LJ, Kuiper P,Wiercinska E, Verspaget HW, Liu Z, Pardali E, et al. Matrix metalloproteinase-14 (MT1-MMP)-mediated endoglin shedding inhibits tumor angiogenesis. Cancer Res. 2010 May;70(10): 4141-50.

20. Venkatesha S, Toporsian M, Lam C, Hanai J, Mammoto T, Kim YM, et al. Soluble endoglin contributes to the pathogenesis of preeclampsia. Nat Med. 2006 Jun;12(6):642-9.

21. Letteboer TG, Zewald RA, Kamping EJ, de Haas G, Mager JJ, Snijder RJ, et al. Hereditary hemorrhagic telangiectasia: ENG and ALK-1 mutations in Dutch patients. Hum Genet. 2005 Jan;116(12):8-16.

22. Lek M, Karczewski KJ, Minikel EV, Samocha KE, Banks E, FennellT, et al.Analysis of protein-coding genetic variation in 60,706 humans. Nature. 2016 Aug;536(7616):285-91.

23. Lenato GM, Lastella P, Di Giacomo MC, Resta N, Suppressa P, Pasculli G, et al. DHPLC-based mutation analysis of ENG and ALK-1 genes in HHT Italian population. Hum Mutat. 2006 Feb;27(2):213-4.

24. McDonald J, Damjanovich K, Millson A, WooderchakW, Chibuk JM,
Stevenson DA, et al. Molecular diagnosis in hereditary hemorrhagic telangiectasia: findings in a series tested simultaneously by sequencing and deletion/duplication analysis. Clin Genet. 2011 Apr;79(4): 335-44.

25. Richards-Yutz J, Grant K, Chao EC, Walther SE, Ganguly A. Update on molecular diagnosis of hereditary hemorrhagic telangiectasia. Hum Genet. 2010 Jul;128(1):61-77.

26. Assis AM, Costa FF, Arruda VR, Annichino-Bizzacchi JM, Bertuzzo CS.Three novel mutations in the activin receptor-like kinase 1 (ALK1) gene in hereditary hemorrhagic telangiectasia type 2 in Brazilian patients. J Hum Genet. 2007;52(3):237-43.

27. Abdalla SA, Gallione CJ, Barst RJ, Horn EM, Knowles JA, Marchuk DA, et al. Primary pulmonary hypertension in families with hereditary haemorrhagic telangiectasia. Eur Respir J. 2004 Mar;23(3): 373-7.

28. Raab U, Velasco B, Lastres P, Letamendia A, Cales C, Langa C, et al. Expression of normal and truncated forms of human endoglin. Biochem J. 1999 May;339(Pt 3):579-88.

29. Berg JN, Gallione CJ, Stenzel TT, Johnson DW, Allen WP, Schwartz $\mathrm{CE}$, et al. The activin receptor-like kinase 1 gene: genomic structure and mutations in hereditary hemorrhagic telangiectasia type 2 . Am J Hum Genet. 1997 Jul;61(1):60-7.

30. Olivieri C, Mira E, Delu G, Pagella F, Zambelli A, Malvezzi L, et al. Identification of 13 new mutations in the ACVRL1 gene in a group of 52 unselected Italian patients affected by hereditary haemorrhagic telangiectasia. J Med Genet. 2002 Jul;39(7):E39.

31. Harrison RE, Flanagan JA, Sankelo M, Abdalla SA, Rowell J, Machado RD, et al. Molecular and functional analysis identifies ALK-1 as the predominant cause of pulmonary hypertension related to hereditary haemorrhagic telangiectasia. J Med Genet. 2003 Dec;40(12): 865-71.

32. Pawlikowska L, Tran MN,Achrol AS, Ha C, Burchard E, Choudhry S, et al. Polymorphisms in transforming growth factor-beta-related genes ALK1 and ENG are associated with sporadic brain arteriovenous malformations. Stroke. 2005 Oct;36(10):2278-80. 